\title{
Uso de cânula rígida com rosca e endoscópio flexível para videolaparoscopia de acesso único em equinos em estação
}

\author{
[Use of threaded rigid cannula and flexible endoscope for single access video laparoscopy \\ in standing horses] \\ L.C. Melo ${ }^{1}$, L.P. Merini ${ }^{1}$, F.U. Bueno ${ }^{1}$, D.E. Loss $^{1}$, D. Zaro ${ }^{1}$, C.A.C. Beck ${ }^{2}$ \\ ${ }^{1}$ Alunos de pós-graduação - Faculdade de Veterinária - Universidade Federal do Rio Grande \\ do Sul - Porto Alegre, RS. \\ ${ }^{2}$ Faculdade de Veterinária - Universidade Federal do Rio Grande do Sul - Porto Alegre, RS
}

\begin{abstract}
RESUMO
Com o objetivo de promover, por meio de acesso único e com o uso de endoscópio flexível, ampla exploração da cavidade peritoneal de equinos em estação, foi concebida uma cânula laparoscópica para dar sustentação ao endoscópio e possibilitar o acesso sob visualização. O procedimento foi realizado a partir da fossa paralombar. Após pequena incisão cutânea, o endoscópio foi inserido na cânula e os músculos e o peritônio foram divulsionados mediante rotação da cânula. Logo depois da perfuração do peritônio, foi realizada a exploração da cavidade e a identificação das estruturas. Em seguida à exploração do lado ipsilateral ao acesso, realizou-se a transposição do conjunto cânula/endoscópio ventralmente à porção caudal do cólon descendente, seguida de exploração do lado contralateral. Concluída a técnica, foi executado, para fins de comparação, o mesmo procedimento por meio da fossa paralombar contralateral. Foi possível a transposição do conjunto cânula/endoscópio para o lado contralateral ao acesso em todos os procedimentos. Também foi possível a identificação da maioria das estruturas abdominais tanto pelo acesso esquerdo quanto pelo direito. A abordagem por acesso único mostrou-se viável para a exploração ampla da cavidade peritoneal, demonstrando ser uma alternativa à técnica laparoscópica convencional.
\end{abstract}

Palavras-chave: cavalos, laparoscopia, endoscópio flexível, cirurgia diagnóstica

\begin{abstract}
A laparoscopic cannula was designed to support a single access approach with a flexible endoscope for the wide exploration of the peritoneal cavity of standing horses. It provides support to the endoscope and allows access to the peritoneal cavity with a visual aid. This procedure was performed through the paralumbar fossa. After a small cutaneous incision, the endoscope was inserted into the cannula, and the muscles and peritoneum were divulsed through the rotation of the cannula. After the peritoneal perforation, cavity exploration and identification of structures were performed. After the exploration of the ipsilateral side of the access, the cannula/endoscope was transposed ventrally to the caudal portion of the descending colon; this was followed by the exploration of the contralateral side. Once this process was completed, the same procedure was performed through the contralateral paralumbar fossa for comparison. It was possible to transpose the cannula/endoscope set to the contralateral access side in all procedures. Further, it was possible to identify most of the abdominal structures in both the left and right access. This single access approach proved to be feasible for the extensive exploration of the peritoneal cavity, thereby indicating it can be an alternative to the conventional laparoscopic technique.
\end{abstract}

Keywords: horses, laparoscopy, flexible endoscope, diagnostic surgery

\section{INTRODUÇÃO}

A laparoscopia diagnóstica é procedimento de grande utilidade em medicina equina, pois fornece informações complementares e, em

Recebido em 9 de janeiro de 2019

Aceito em 1 de outubro de 2019

E-mail: lucianovt@gmail.com alguns casos, o diagnóstico definitivo e o prognóstico de doenças abdominais. Vários trabalhos das áreas médica e veterinária destacam que a maioria das complicações que ocorrem durante a laparoscopia estão relacionadas à inserção do primeiro trocater 
(Ternamian, 1998; Walmsley, 2007; Djokovic et al., 2016). Entre as complicações mais comumente relatadas na laparoscopia em equinos em estação estão o descolamento peritoneal, as lesões no baço, a punção intestinal e as lesões em vasos sanguíneos da parede abdominal (Walmsley, 1999; Desmaizières et al., 2003).

Com o intuito de promover segurança ao acesso laparoscópico, foi desenvolvida a cânula óptica com rosca sem obturador EndoTIP ${ }^{\mathrm{TM}}$ (Karl Storz), cujo protótipo foi descrito por Ternamian (1997). Essa cânula possui um tubo oco de aço inoxidável, apresentando externamente uma rosca de perfil positivo que termina distalmente em uma ponta romba. Dessa forma, o acesso laparoscópico é realizado sob visualização, uma vez que um laparoscópio com ângulo de visão de $0^{\circ}$ é introduzido no interior da cânula, substituindo o obturador. À medida que a força de rotação é aplicada, a rosca externa promove a divulsão proximal das sucessivas camadas de tecidos da parede abdominal, até que a cânula atinja a cavidade peritoneal com segurança e sob controle visual (Ternamian, 1998).

A técnica convencional de laparoscopia diagnóstica com o equino em estação e abordagem por meio da fossa paralombar exige que sejam realizados dois procedimentos laparoscópicos (um de cada lado do animal) para visualização significativa da cavidade peritoneal, além do uso de laparoscópio rígido (Galuppo et al., 1995; Silva et al., 2008). A utilização de endoscópio flexível (longo, maleável e amplamente disponível em clínicas e hospitais para equinos), somada ao desenvolvimento de novas técnicas e instrumentos que permitam visualizar ambos os lados do abdome por um único acesso, poderá tornar o procedimento mais simples e rápido, reduzir os custos e popularizar a técnica, podendo representar um avanço para a laparoscopia na espécie equina.

Com o objetivo de promover sustentação e estabilidade ao endoscópio flexível e favorecer o acesso seguro à cavidade peritoneal através de rosca inspirada na cânula EndoTIP ${ }^{\mathrm{TM}}$, foi desenvolvida a cânula laparoscópica deste estudo. Assim, este trabalho tem por objetivo avaliar a viabilidade do procedimento videolaparoscópico por acesso único via fossa paralombar direita e esquerda, utilizando endoscópio flexível inserido através dessa cânula na exploração e identificação das estruturas da cavidade peritoneal de equinos em estação.

\section{MATERIAL E MÉTODOS}

Este estudo foi avaliado pela Comissão de Ética no Uso de Animais da Universidade Federal do Rio Grande do Sul, sendo aprovado sob o número 27639.

Foram utilizados seis equinos adultos (três machos e três fêmeas), hígidos, sem raça definida, com idade entre quatro e 20 anos e massa corpórea entre $260 \mathrm{~kg}$ e $380 \mathrm{~kg}$.

A cânula laparoscópica concebida para este estudo (projeto em parceria com a Bhio Supply, Brasil) apresenta, na porção proximal, um sistema de válvulas onde há uma torneira para insuflação de gases, um diafragma com acionamento manual e uma borracha de vedação, seguida por um tubo oco de aço inoxidável de $60 \mathrm{~cm}$ de comprimento e $16 \mathrm{~mm}$ de diâmetro. $\mathrm{Na}$ extremidade distal da cânula, há externamente uma rosca de perfil positivo de $4 \mathrm{~cm}$ de comprimento, que termina distalmente em uma ponta romba (Fig.1).

O endoscópio flexível (Pentax EC-380IL, 160cm x 12mm, Pentax Corporation, Japão) e a borracha de vedação da cânula laparoscópica foram submetidos à desinfecção de alto nível por submersão em solução de glutaraldeído $2 \%$, durante 45 minutos. Posteriormente, o endoscópio foi protegido com capa plástica videoendoscópica estéril. A cânula laparoscópica e o instrumental cirúrgico convencional foram esterilizados por autoclavagem.

Os equinos foram submetidos a jejum alimentar de 24 a 30 horas e permaneceram com água $a d$ libitum até cerca de duas horas antes do procedimento. No bloco cirúrgico, os animais foram posicionados em tronco de contenção, sendo mantidos em estação durante todo o procedimento cirúrgico. $\mathrm{O}$ reto foi esvaziado manualmente e as regiões a serem acessadas foram avaliadas por palpação via transretal. Apesar de a técnica proposta ser o acesso único, em caráter experimental, foi realizado o acesso através de ambas as fossas paralombares do mesmo animal, com o intuito de comparação. 


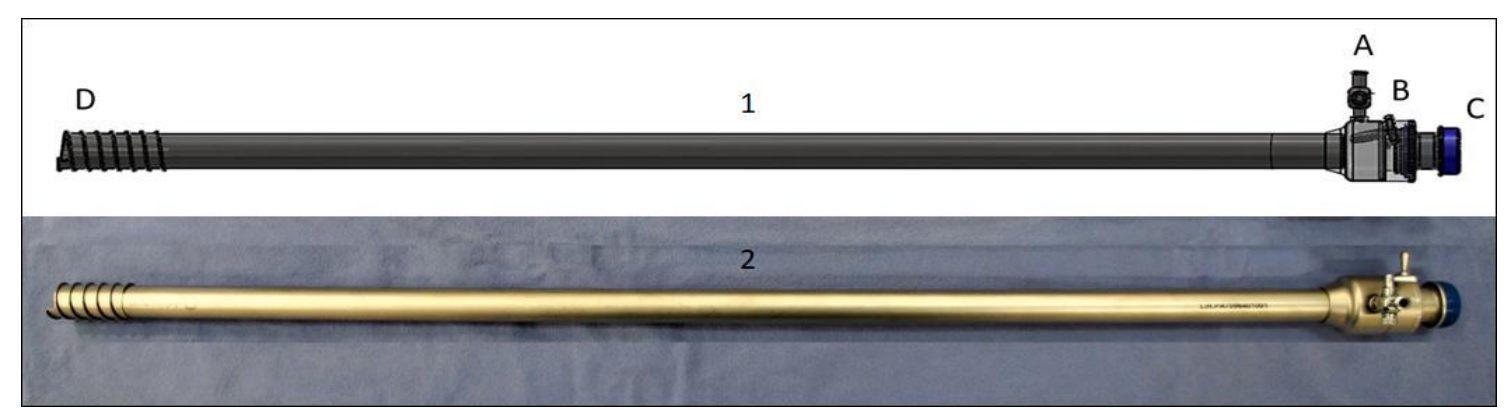

Figura 1. Desenho esquemático (1) e imagem (2) da cânula laparoscópica (cânula rígida com rosca sem obturador) de $60 \mathrm{~cm}$ de comprimento utilizada neste estudo. (A) torneira para insuflação de gases; (B) diafragma com acionamento manual; (C) borracha de vedação; (D) rosca de perfil positivo de $4 \mathrm{~cm}$ de comprimento.

Os equinos foram sedados com cloridrato de detomidina $(0,02 \mathrm{mg} / \mathrm{kg}$ IV). Logo após a tricotomia, a antissepsia cirúrgica e a fixação do campo cirúrgico, foi realizado o bloqueio anestésico local com $20 \mathrm{~mL}$ de cloridrato de lidocaína $2 \%$ infundida na musculatura e tecido subcutâneo do local de incisão, no centro da linha entre a borda caudal da última costela e a borda ventral da tuberosidade coxal. O procedimento cirúrgico teve início com uma pequena incisão cutânea de cerca de dois centímetros de comprimento no centro da fossa paralombar direita ou esquerda (o primeiro acesso foi determinado por sorteio). As camadas musculares da região e o peritônio foram divulsionados por meio da rotação da cânula laparoscópica em sentido horário e a divulsão dos tecidos foi acompanhada pelas imagens produzidas pelo endoscópio flexível inserido na cânula e reproduzidas no monitor (Fig. 2). A insuflação positiva de gases para a obtenção do pneumoperitônio não foi instituída. $\mathrm{O}$ abdome equino foi didaticamente dividido em quatro quadrantes: cranial direito, caudal direito, cranial esquerdo e caudal esquerdo. O conjunto cânula laparoscópica e extremidade livre do endoscópio foi movimentado pelo cirurgião, e os comandos do endoscópio controlados pelo endoscopista.

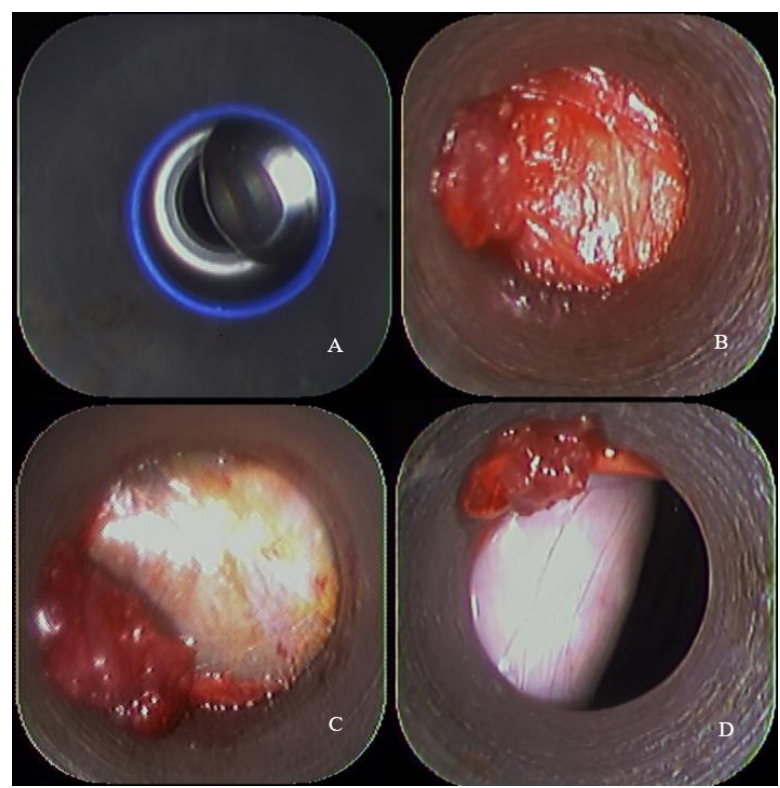

Figura 2. Imagens obtidas pelo endoscópio flexível posicionado no interior da cânula rígida com rosca durante as etapas de acesso laparoscópico na fossa paralombar de equino em estação, por meio de movimento rotacional. (A) abertura manual do diafragma da cânula, (B) divulsão muscular, (C) divulsão do peritônio, (D) acesso à cavidade peritoneal. 
O exame exploratório teve início após a penetração do conjunto cânula/endoscópio na cavidade peritoneal (Fig. 3). Em seguida, o conjunto foi direcionado cranialmente, $\mathrm{e}$ as estruturas da região identificadas. Após a exploração da região cranial do lado ipsilateral ao acesso, o conjunto foi dirigido caudalmente para a exploração dessa região. Finalizando essa etapa, a transposição do conjunto cânula/endoscópio para o lado contralateral foi realizada entre os quadrantes caudais, ventralmente à porção caudal do cólon descendente, medialmente ao anel inguinal interno (nos machos), ou ventralmente ao corno uterino, nas fêmeas (Fig. 4). Após a transposição, movia-se principalmente o endoscópio. As estruturas caudais do lado contralateral foram primeiramente identificadas, seguidas pela identificação das estruturas craniais. As estruturas visualizadas foram identificadas por suas características morfológicas e situação topográfica e registradas em fichas específicas.

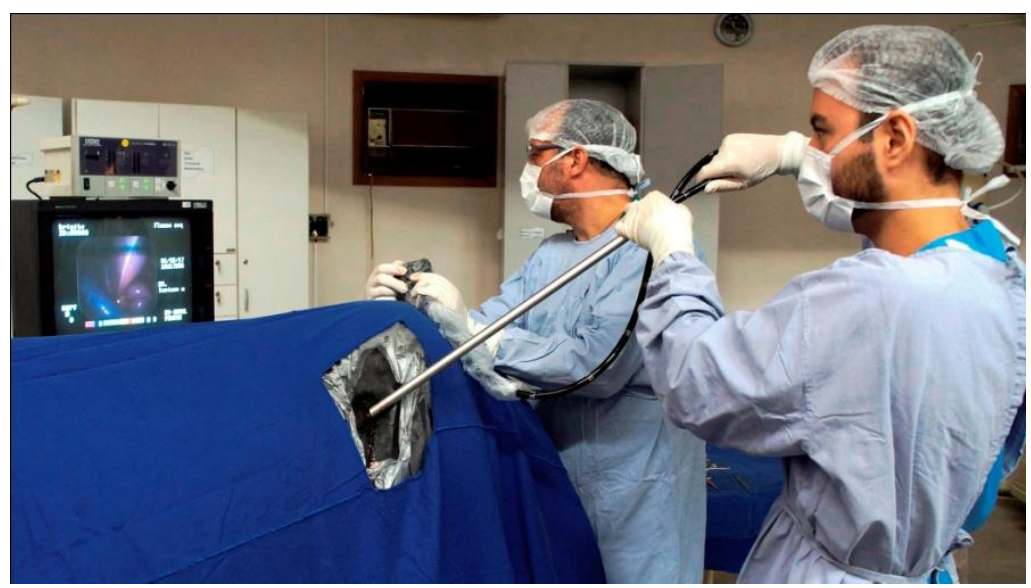

Figura 3. Início da exploração videolaparoscópica da cavidade peritoneal de equino em estação via fossa paralombar, com a utilização de cânula rígida com rosca e endoscópio flexível.

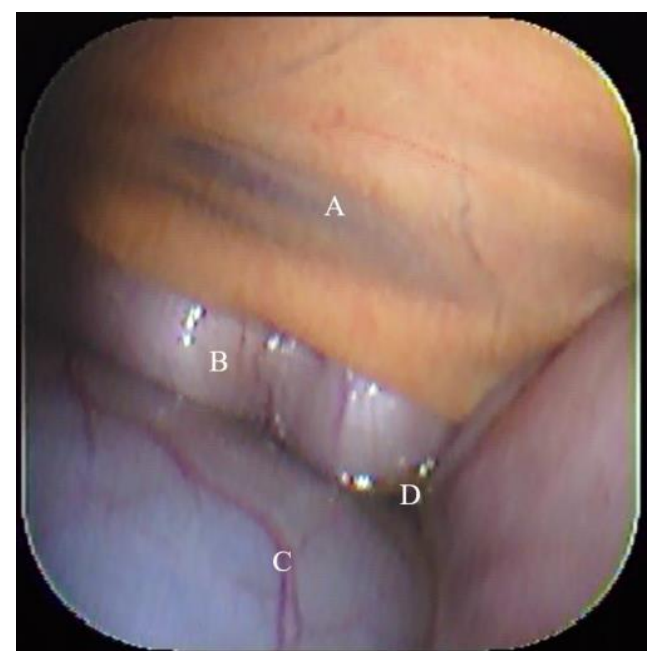

Figura 4. Imagem videolaparoscópica da região caudal direita da cavidade peritoneal de um equino em estação. (A) mesocólon do cólon descendente, (B) cólon descendente, (C) bexiga, (D) local de transposição do conjunto cânula/endoscópio para a esquerda.

Ao final da exploração, o endoscópio e a cânula foram removidos por meio da rotação da cânula no sentido anti-horário. A incisão de pele foi suturada junto ao subcutâneo em padrão isolado simples, utilizando-se fio de náilon monofilamentar número zero. Imediatamente após a sutura, a posição da torre de equipamentos e da mesa com instrumental cirúrgico foi 
invertida, e o procedimento descrito realizado na fossa paralombar contralateral.

Foi realizada profilaxia antitetânica com 5.000UI/animal (IM) de antitoxinas tetânicas previamente ao procedimento. Como terapia analgésica e anti-inflamatória, os animais receberam flunixina meglumina $(1,1 \mathrm{mg} / \mathrm{kg}$ IV) previamente ao início do procedimento e a cada 24 horas, totalizando três aplicações. Como terapia antimicrobiana, foi utilizada associação de penicilinas e estreptomicinas (benzilpenicilina benzatina, benzilpenicilina procaína, benzilpenicilina potássica, sulfato de diidroestreptomicina e sulfato de estreptomicina), na dose de $30.000 \mathrm{UI} / \mathrm{kg}$ IM (calculada sobre a concentração de penicilina benzatina), duas horas antes do procedimento e 48 horas após. Exame clínico geral foi realizado diariamente até a retirada das suturas, seguido por inspeção e higienização do local das incisões. As suturas cutâneas foram retiradas no $10^{\circ}$ dia após a cirurgia.

\section{RESULTADOS E DISCUSSÃO}

O jejum alimentar pré-cirúrgico de 24 a 30 horas mostrou-se suficiente para o esvaziamento regular do trato gastrintestinal, permitindo a visualização das estruturas. No entanto, foram perceptíveis pequenas variações entre os animais quanto à presença de conteúdo intestinal, o que influenciou na maior ou menor dificuldade de exploração. Diante disso, é possível sugerir que jejum mínimo de 24 horas seja adotado e que, se possível, período maior seja realizado. Segundo Hendrickson (2009), o trato digestório menos distendido permite uma melhor avaliação dos órgãos abdominais e do peritônio e menor risco de iatrogenia ao introduzir a primeira cânula.

Neste trabalho, a insuflação com pressão positiva para a obtenção do pneumoperitônio não foi instituída. Após romper o peritônio, a pressão negativa da cavidade peritoneal era desfeita e o ar ambiente penetrava naturalmente através da cânula, promovendo espaço suficiente para a visualização das estruturas. Da mesma forma, Alford e Hanson (2010) descreveram que a insuflação do abdome de equinos com pressão positiva não foi utilizada ou considerada necessária para se obter uma visualização adequada em procedimentos laparoscópicos por colpotomia. Walmsley (2007) descreve que, em sua experiência, a insuflação positiva é raramente necessária na laparoscopia de equinos em estação.

A divulsão das camadas musculares e do peritônio mediante a inserção da cânula foi realizada sem dificuldade. A rosca na extremidade da cânula permitiu sua inserção até a cavidade peritoneal a partir de sua rotação, promovendo a divulsão dos tecidos, enquanto o endoscópio forneceu as imagens reproduzidas no monitor para que o acesso fosse realizado sob controle visual. $\mathrm{O}$ acesso laparoscópico obtido com a cânula mostrou-se seguro e corrobora a descrição de Ternamian (1998) de que as cânulas laparoscópicas com rosca e sem obturador convertem a força de entrada linear, excessiva e não controlada em uma força de rotação radial e permitem o acesso sob visualização. Em nenhum animal houve lesão a vasos sanguíneos, desprendimento peritoneal ou punção intestinal.

No entanto, em dois animais houve pequena lesão iatrogênica ao baço, evidenciada por discreta hemorragia local. Uma das complicações associadas à laparoscopia em equinos em estação é a ocorrência de punção esplênica no momento da inserção da primeira cânula (Desmaizières $e t$ al., 2003; Hendrickson, 2009; Huppes et al., 2017), fato que ocorreu em um dos equinos. Provavelmente a falta de experiência pode ter contribuído para a iatrogenia, pois esse animal foi o primeiro a ser submetido ao procedimento do estudo. No segundo animal, a lesão esplênica ocorreu após a transposição do conjunto cânula/endoscópio da direita para a esquerda, pelo contato da extremidade da cânula com a superfície do baço. Apesar da inocuidade dessa lesão, o fato chama a atenção para que sejam realizadas adequações no design da cânula visando à maior segurança em seu uso. Da mesma forma que descrito por Walmsley (1999) e Hendrickson (2009), as lesões esplênicas provocadas neste estudo foram semelhantes a uma biópsia, tendo ambas alcançado resolução espontânea sem necessidade de tratamento.

A utilização da cânula promoveu a estabilidade e a sustentação necessárias ao endoscópio para avançá-lo até pontos mais distantes do local de penetração e permitiu a exploração abdominal tanto ipsilateral como do lado contralateral. Em todos os animais foi possível realizar a transposição do conjunto cânula/endoscópio para 
o lado oposto. Sua movimentação entre os órgãos abdominais mostrou-se segura, demonstrando ser eficiente quando associada ao endoscópio flexível para procedimentos laparoscópicos exploratórios por acesso único. Tal condição merece destaque, pois a possibilidade de exploração de ambos os lados do abdome por um único ponto de acesso traz uma inovação importante para as técnicas laparoscópicas descritas em equinos, reduzindo tempo operatório e custos.

O local de transposição do conjunto cânula/endoscópio ventralmente à porção caudal do cólon descendente e tomando como referência $\mathrm{o}$ anel inguinal interno (nos machos) e o corno uterino (nas fêmeas) se deveu ao fato de não haver inúmeras dobras mesentéricas cobrindo essa região, como ocorre nas porções mais craniais. Tecnicamente, esse local parece ser o único possível onde realizar a transposição.

O conjunto cânula/endoscópio foi movimentado pelo cirurgião, e os comandos do endoscópio controlados pelo endoscopista. Apesar da necessidade de dois cirurgiões, a abordagem em equipe permitiu uma técnica cirúrgica eficiente, pois, enquanto o cirurgião manipulava a cânula e o endoscópio, o endoscopista controlava o ângulo de visão, corroborando Alford e Hanson (2010), que descreveram maior eficiência na abordagem em equipe quando se utiliza um endoscópio flexível.

As estruturas abdominais visualizadas estão listadas nas Tab. 1 e 2 . Foi possível a identificação da maioria das estruturas abdominais descritas na literatura por procedimento laparoscópico em equinos em estação (Galuppo et al., 1995; Trostle, 2000; Alford e Hanson, 2010; Nóbrega et al., 2011). Vale ressaltar que as estruturas observadas durante o exame laparoscópico podem variar dependendo do porte e do posicionamento do animal, do local de acesso, das variações fisiológicas (Silva et al., 2008), da técnica e dos equipamentos

Tabela 1. Número e porcentagem de visualizações de estruturas anatômicas localizadas na cavidade peritoneal esquerda de equinos submetidos a procedimento videolaparoscópico em estação por acesso via fossa paralombar esquerda e direita, utilizando endoscópio flexível e cânula laparoscópica de $60 \mathrm{~cm}$ de comprimento $(\mathrm{n}=6)$.

\begin{tabular}{llllll}
\hline Região & Estrutura anatômica esquerda & \multicolumn{2}{l}{ Acesso ipsilateral } & \multicolumn{2}{l}{ Acesso contralateral } \\
\hline \multirow{2}{*}{ Cranial } & Diafragma & 6 & $100 \%$ & 6 & $100 \%$ \\
& Lobo hepático lateral esquerdo & 6 & $100 \%$ & 6 & $100 \%$ \\
& Ligamento triangular esquerdo & 6 & $100 \%$ & 6 & $100 \%$ \\
& Ligamento gastrofrênico & 6 & $100 \%$ & 4 & $66,6 \%$ \\
& Estômago & 6 & $100 \%$ & 6 & $100 \%$ \\
& Baço & 6 & $100 \%$ & 6 & $100 \%$ \\
& Ligamento gastroesplênico & 3 & $50 \%$ & 2 & $33,3 \%$ \\
& Ligamento frenicoesplênico & 6 & $100 \%$ & 1 & $16,6 \%$ \\
& Ligamento nefroesplênico & 6 & $100 \%$ & 6 & $100 \%$ \\
Rim esquerdo & 6 & $100 \%$ & 5 & $83,3 \%$ \\
& Líquido peritoneal & 6 & $100 \%$ & 6 & $100 \%$ \\
Jejuno e íleo - segmentos & 6 & $100 \%$ & 6 & $100 \%$ \\
& Cólon descendente - segmentos & 6 & $100 \%$ & 6 & $100 \%$ \\
Mesocólon cólon descendente & 6 & $100 \%$ & 6 & $100 \%$ \\
Cólon dorsal esquerdo & 0 & $0 \%$ & 0 & $0 \%$ \\
Flexura pélvica & 0 & $0 \%$ & 0 & $0 \%$ \\
Reto & 5 & $83,3 \%$ & 0 & $0 \%$ \\
Bexiga urinária e lig. lateral & 4 & $66,6 \%$ & 1 & $16,6 \%$ \\
Parede abdominal esquerda & 6 & $100 \%$ & 6 & $100 \%$ \\
Cordão espermático & 3 & $100 \%$ & 3 & $100 \%$ \\
Anel inguinal interno & 2 & $66,6 \%$ & 2 & $66,6 \%$ \\
Ovário esquerdo e ligamentos & 3 & $100 \%$ & 2 & $66,6 \%$ \\
Corno uterino esquerdo e lig. & 3 & $100 \%$ & 2 & $66,6 \%$ \\
\hline
\end{tabular}


Tabela 2. Número e porcentagem de visualizações de estruturas anatômicas localizadas na cavidade peritoneal direita de equinos submetidos a procedimento videolaparoscópico em estação por acesso via fossa paralombar esquerda e direita, utilizando endoscópio flexível e cânula laparoscópica de $60 \mathrm{~cm}$ de comprimento $(n=6)$.

\begin{tabular}{|c|c|c|c|c|c|}
\hline \multirow[t]{2}{*}{ Região } & \multirow[t]{2}{*}{ Estrutura anatômica direita } & \multicolumn{2}{|c|}{ Acesso ipsilateral } & \multicolumn{2}{|c|}{ Acesso contralateral } \\
\hline & & $\mathrm{N}^{\circ}$ & $\%$ & $\mathrm{~N}^{\mathrm{o}}$ & $\%$ \\
\hline \multirow[t]{14}{*}{ Cranial } & Diafragma & 4 & $66,6 \%$ & 2 & $33,3 \%$ \\
\hline & Lobo hepático direito & 6 & $100 \%$ & 6 & $100 \%$ \\
\hline & Ligamento triangular direito & 6 & $100 \%$ & 5 & $83,3 \%$ \\
\hline & Cólon dorsal direito & 6 & $100 \%$ & 6 & $100 \%$ \\
\hline & Processo caudado & 6 & $100 \%$ & 6 & $100 \%$ \\
\hline & Forame epiploico & 6 & $100 \%$ & 6 & $100 \%$ \\
\hline & Pâncreas & 3 & $50 \%$ & 1 & $16,6 \%$ \\
\hline & Rim direito & 6 & $100 \%$ & 6 & $100 \%$ \\
\hline & Ligamento hepatorrenal & 6 & $100 \%$ & 6 & $100 \%$ \\
\hline & Duodeno - porção descendente & 6 & $100 \%$ & 6 & $100 \%$ \\
\hline & Duodeno - flexura caudal & 6 & $100 \%$ & 4 & $66,6 \%$ \\
\hline & Duodeno - porção ascendente & 6 & $100 \%$ & 0 & $0 \%$ \\
\hline & Mesoduodeno & 6 & $100 \%$ & 6 & $100 \%$ \\
\hline & Líquido peritoneal & 6 & $100 \%$ & 6 & $100 \%$ \\
\hline \multirow[t]{12}{*}{ Caudal } & Ceco - base & 6 & $100 \%$ & 6 & $100 \%$ \\
\hline & Cólon ventral direito & 4 & $66,6 \%$ & 2 & $33,3 \%$ \\
\hline & Jejuno e íleo - segmentos & 6 & $100 \%$ & 4 & $66,6 \%$ \\
\hline & Cólon descendente - segmentos & 6 & $100 \%$ & 3 & $50 \%$ \\
\hline & Mesocólon cólon descendente & 6 & $100 \%$ & 3 & $50 \%$ \\
\hline & Reto & 6 & $100 \%$ & 1 & $16,6 \%$ \\
\hline & Bexiga urinária e lig. lateral & 5 & $83,3 \%$ & 1 & $16,6 \%$ \\
\hline & Parede abdominal direita & 6 & $100 \%$ & 6 & $100 \%$ \\
\hline & Cordão espermático & 3 & $100 \%$ & 3 & $100 \%$ \\
\hline & Anel inguinal interno & 3 & $100 \%$ & 3 & $100 \%$ \\
\hline & Ovário direito e ligamentos & 3 & $100 \%$ & 3 & $100 \%$ \\
\hline & Corno uterino direito e lig. & 3 & $100 \%$ & 3 & $100 \%$ \\
\hline
\end{tabular}

O cólon dorsal esquerdo e a flexura pélvica não foram visualizados em nenhum animal. Segundo Silva et al. (2008), a flexura pélvica e as porções do cólon ventral e dorsal esquerdos podem ser eventualmente identificadas por laparoscopia em estação, mas essas estruturas normalmente são cobertas por segmentos de intestino delgado ou cólon descendente, especialmente após jejum.

Foi perceptível maior grau de dificuldade na exploração contralateral ao acesso, pois, após a transposição do conjunto cânula/endoscópio, havia uma limitação na movimentação da cânula e a exploração foi realizada principalmente pela movimentação do endoscópio, resultando em maior instabilidade devido a sua flexibilidade. Assim, algumas estruturas não foram identificadas em alguns animais após a transposição do conjunto cânula/endoscópio da mesma forma em que foram identificadas pelo acesso ipsilateral. Apesar dessas dificuldades, a técnica proposta mostrou-se promissora para que a exploração da cavidade peritoneal por acesso único seja semelhante à obtida pela técnica convencional de acesso por ambos os lados do abdome.

Não foram observadas alterações nos exames clínicos pós-cirúrgicos, e, em todos os animais, a cicatrização das feridas cirúrgicas evoluiu sem intercorrências.

\section{CONCLUSÃO}

De acordo com os resultados obtidos e nas condições em que foi realizado o presente estudo, pode-se concluir que: o procedimento videolaparoscópico por acesso único via fossa paralombar esquerda e via fossa paralombar direita, utilizando endoscópio flexível inserido 
através da cânula laparoscópica proposta na exploração e identificação de estruturas da cavidade peritoneal é viável; a cânula com rosca se mostrou adequada ao uso, demonstrando ser segura para o acesso laparoscópico e para a transposição e exploração do lado contralateral ao acesso; e não foram observadas diferenças quanto a vantagens ou dificuldades entre o acesso via fossa paralombar esquerda e via fossa paralombar direita para identificar as estruturas ipsilaterais e contralaterais correspondentes.

\section{REFERÊNCIAS}

ALFORD, C.; HANSON, R. Evaluation of a transvaginal laparoscopic natural orifice transluminal endoscopic surgery approach to the abdomen of mares. Vet. Surg., v.39, p.873-878, 2010 .

DESMAIZIÈRES, L.M.; MARTINOT, S.; LEPAGE, O.M. et al. Complications associated with cannula insertion techniques used for laparoscopy in standing horses. Vet. Surg., v.32, p.501-506, 2003.

DJOKOVIC, D.; GUPTA, J.; THOMAS, V. et al. Principles of safe laparoscopic entry. Eur. J. Obstet. Gynecol. Reprod. Biol., v.201, p.179188, 2016.

GALUPPO, L.D.; SNYDER, J.R.; PASCOE, J.R. Laparoscopic anatomy of the equine abdomen. Am. J. Vet. Res., v.56, p.518-531, 1995.
HENDRICKSON, D.A. Complications of laparoscopic surgery. Vet. Clin. N. Am. Equine Pract., v.24, p.557-571, 2009.

HUPPES, T.; STOUT, T.A.E.; ENSINK, J.M. Decision making for cryptorchid castration; a retrospective analysis of 280 cases. J. Equine Vet. Sci., v.48, p.73-81, 2017.

NÓBREGA, F.S.; BECK, C.A.C.; FERREIRA M.P. et al. Videolaparoscopia topográfica de equinos em estação com três diferentes massas corpóreas. Arq. Bras. Med. Vet. Zootec., v.63, p.873-882, 2011.

SILVA, L.C.L.C.; ZOPPA, A.L.V.; HENDRICKSON, D.A. Equine diagnostic laparoscopy. J. Equine Vet. Sci., v.28, p.247-254, 2008.

TERNAMIAN, A.M. A trocarless, reusable, visual-access cannula for safer laparoscopy; an update. J. Am. Assoc. Gynecol. Laparosc., v.5, p.197-201, 1998.

TERNAMIAN, A.M. Laparoscopy without trocars. Surg. Endosc., v.11, p.815-818, 1997.

TROSTLE, S. Gastrointestinal endoscopic surgery. Vet. Clin. North. Am. Equine Pract., v.16, p.329-341, 2000.

WALMSLEY, J.P. Laparoscopy in horses with abdominal pain. Equine Vet. Educ., v.19, p.6466, 2007.

WALMSLEY, J.P. Review of equine laparoscopy and an analysis of 158 laparoscopies in the horse. Equine Vet. J., v.31, p.456-464, 1999. 